\title{
THE PHOTODISINTEGRATION OF COSMIC RAY NUCLEI BY SOLAR PHOTONS: THE GERASIMOVA-ZATSEPIN EFFECT REVISITED
}

\author{
Gustavo A. Medina-Tanco ${ }^{1,2}$ \\ and \\ Alan A. Watson ${ }^{2}$ \\ 1. Instituto Astronômico e Geofísico, University of São Paulo, Brasil \\ gustavo@iagusp.usp.br \\ 2. Dept. of Physics and Astronomy, University of Leeds, Leeds LS2 9JT, UK \\ a.a.watson@leeds.ac.uk
}

Received —; accepted $\longrightarrow$ 


\begin{abstract}
The interesting possibility of measuring the masses of high energy cosmic ray particles by observing pairs of extensive air showers arriving at the earth nearly simultaneously was proposed some years ago by Zatsepin (1951) and Gerasimova and Zatsepin (1960). Such showers would be created by the nuclear fragments originating as a result of the photodisintegration of massive nuclei interacting with the solar radiation field. In this paper we re-visit this possibility in the context of existing and proposed detectors of high and ultra-high energy cosmic rays considering a simple, yet realistic, model of the interplanetary magnetic field. The possibility of observing the mass fragmentation of cosmic rays directly, however, remains challenging.

Subject headings: cosmic rays — interplanetary magnetic field photodisintegration — extensive air showers
\end{abstract}




\section{Introduction}

Cosmic ray nuclei travelling towards the Earth can interact with the solar radiation field and photodisintegrate (Zatsepin, 1951), leading to the almost simultaneous creation of pairs of extensive air showers (EAS) in the earth's atmosphere. If such pairs of showers could be detected and the initiating energies measured then the ratio of the greater energy to the lesser energy would give directly the mass of the heavier fragment. This assumes, as is most probable, that photodisintegration produces a single nucleon as one of the fragments. Thus in principle the mass of incoming cosmic rays could be measured rather directly. Gerasimova and Zatsepin (1960) studied this phenomenon in a simplified analytical way which was made necessary by the complexity of the problem and the limited computing power then available. As the problem of the mass composition of cosmic rays above the knee of the spectrum remains as important and controversial an issue as it was in the sixties (e.g., Watson 1997) it is of interest to assess the potential of any mechanism that could help to tackle the problem. With its remarkable simplicity, the Gerasimova-Zatsepin mechanism certainly falls in this category.

Our main contribution is to address an important issue which was treated incompletely in the initial work namely the effect of the interplanetary magnetic field on the distribution of the expected core separations. We have made a significant improvement in this regard by performing exact orbit integrations in a realistic model of the interplanetary magnetic field. We also use modern estimates of the primary energy spectrum to calculate the expected rate of arrival of such pairs of showers and discuss the possibility of detection with current and planned instruments.

The relevant photonuclear interactions are discussed, for example, in Danos and Fuller (1965), Hayward (1970) or Puget, Stecker and Bredekamp (1976). The energy range of interest in the rest frame of the cosmic ray nucleus spans from approximately $10 \mathrm{MeV}$ up 
to $150 \mathrm{MeV}$. However, it is in the region from $\sim 10 \mathrm{MeV}$ to $\sim 30 \mathrm{MeV}$, the domain of the giant resonances of the nuclear photoeffect, where most of the interactions result in the emission of single nucleons, although two-nucleon emission can also occur but with much reduced probability.

In the next section we describe our calculations and assumptions and discuss the results, while our brief concluding remarks are left to the last section.

\section{Numerical calculations and discussion of results}

In dealing with this problem, two different aspects must be considered: the expected event rate and the relative deflection of the fragments.

In the original paper (Gerasimova and Zatsepin 1960) a deviation of the order of $10^{-2}$ $\mathrm{cm}$ is quoted for an assumed homogeneous interplanetary field of the order of $10^{-5}$ Gauss. This value is in error by several orders of magnitude for a typical particle with $\gamma \sim 10^{7}$ interacting with a photon in the vicinity of the Sun. An elementary calculation shows that deflections of hundreds of kilometers should be expected at Earth for a $\gamma \sim 10^{7} \mathrm{Fe}$ nucleus losing a proton by photodisintegration at $1 \mathrm{AU}$. The oversight was subsequently recognized by Zatsepin, as reported by Ginzburg and Syrovatsky (1964), but no detailed calculations seem to have been made subsequently which include the effects of deflections in the interplanetary magnetic field.

For our calculations we use a realistic, yet simple, model of the interplanetary magnetic field (IPMF) due to Akasofu, Gray and Lee (1980). This model takes into account four different components to describe the spiral 3-dimensional structure of the IPMF inside the central 20 AU of the Solar System: (1) the dipole component (B dipole), (2) the sunspot 
component ( $\left.\mathbf{B}_{\text {sunspot }}\right)$, (3) the dynamo component $\left(\mathbf{B}_{\mathbf{d y n a m o}}\right)$ and the ring current component $\left(\mathbf{B}_{\text {ring }}\right)$. The total field is thus expressed as:

$$
\vec{B}=\vec{B}_{\text {dipole }}+\vec{B}_{\text {sunspot }}+\vec{B}_{\text {dynamo }}+\vec{B}_{\text {ring }}
$$

The solar dipole moment is well known $\left(\sim 3.4 \times 10^{-32}\right.$ Gauss $\left.\mathrm{cm}^{3}\right)$. The dynamo component originates in a poloidal current system which exits the Sun at its poles, reaches the heliosphere at high latitudes, flows over this surface towards the ecliptic plane and finally closes the circuit through an inward equatorial current. This system is supposed to be generated by a dynamo process induced by the solar rotation in the dipolar field. The ring current component is produced by a thin equatorial sheet current that extends up to the heliopause. The sunspot component constitutes the magnetic arcade immediately above the photosphere. In the model of Akasofu, Gray and Lee (1980) this component is represented by an ensemble of spherical dipoles just below the surface. Its main purpose is to allow the connection of all the field lines of the equatorial sheet to the Sun's surface without significantly distorting the solar dipole field. Both the solar dipolar component and the sunspot component decrease as $\propto r^{-3}$ and therefore, outside the coronal region, the IPMF is mainly determined by the dynamo and ring current contributions. In figure 1a we show the magnitudes of the total IPMF, and of the ring and dynamo components as a function of the cylindrical coordinate $\rho$ over a plane located at $\mathrm{z}=0.01 \mathrm{AU}$ above the ecliptic. Figure 1b shows the corresponding cylindrical components of the IPMF. The signs over the curves indicate the sign of each component. Note, however, that all the components of the magnetic field reverse their direction with the 11 year solar cycle.

The interplanetary medium is permeated by this magnetic field and by the photon radiation field originating at the Sun's photosphere. For our purpose it is sufficient to assume that the radiation field spectrum is that of a black body at $T_{\text {eff }}=5770 \mathrm{~K}$. 
Consequently, the number density of photons at a distance $r_{A U}$ (in astronomical units) from the Sun is:

$$
n\left(\epsilon_{e V}\right) d \epsilon_{e V} \sim 7.8 \times 10^{7} \times \frac{1}{r_{A U}^{2}} \times \frac{\epsilon_{e V}^{2} d \epsilon_{e V}}{\exp \left(\epsilon_{e V} / 0.5\right)-1} \quad\left[\mathrm{~cm}^{-3}\right]
$$

where $\epsilon_{e V}$ is the energy of the photons in $\mathrm{eV}$ in the reference system of the Sun.

Every cosmic ray particle penetrating the Solar System must traverse this environment before reaching the Earth. In the rest frame of the nucleus the energy of the photons is boosted to:

$$
\epsilon_{N}=\epsilon\left(\gamma+\sqrt{\gamma^{2}-1} \cos \alpha\right) \sim 2 \gamma \epsilon \cos ^{2} \frac{\alpha}{2}
$$

where $\alpha$ is the angle between the momenta of photon and nucleus in the Sun's reference system (e.g. Gerasimova and Zatsepin 1960). When the energy of the photons in the frame of the nuclei is larger than some few $\mathrm{MeV}$, the nuclei can undergo photodisintegration. This process is most important between 15 and $25 \mathrm{MeV}$, in the region of the peak of the giant dipole resonance, although there is still a significant contribution to the cross section from energies beyond $25 \mathrm{MeV}$ up to the threshold for photo-pion production at $\sim 145 \mathrm{MeV}$ (Puget, Stecker and Bredekamp 1976, Hillas, 1975). In the lower energy band, i.e. in the region of the giant dipole resonance (say, $\epsilon \widetilde{<} 30 \mathrm{MeV}$ ), mainly single nucleons are emitted although double nucleon emission also takes place. At higher energies non-resonance processes are responsible for multinucleon emission. The corresponding cross-section parameters and branching ratios can be found in Puget, Stecker and Bredekamp (1976). Gerasimova and Zatsepin (1960) use a form for the cross-section that includes only the giant dipole resonance. We use the Gerasimova and Zatsepin representation of the cross-section at low energies, but have included additionally the non-resonant contribution 
at higher energies up to the photo-pion production threshold. In Figure 2 we compare the cross-sections for Fe as calculated by Gerasimova an Zatsepin (1960), Puget, Stecker and Bredekamp (1976), an approximation by Hillas (1975), and the cross section used in the present work. It is evident that, although the Gerasimova and Zatsepin cross section is wider than the Lorentzian function used in Hillas's approximation, it compares rather well with the combination of single and double nucleon emission as given by Puget and coworkers. Our approximation should give an upper limit to the photodisintegration rate. Uncertainties in the photodisintegration cross section will translate to only some few $10 \%$ and this does not alter our conclusion significantly.

The emission of the nucleon(s) can be assumed to be isotropic in the reference system of the nucleus. Transforming to the Earth reference system, the emission of the fragments is concentrated within a cone of aperture $\sim 1 / \gamma$ around the original direction of propagation of the parent nucleus. Therefore, at the high Lorentz factors of interest here $\left(\gamma>10^{7}\right)$, we can assume that both fragments have, after the interaction with the photon, exactly the same direction as the incoming nucleus. Hence, we calculate relative deflections solely as the product of the differential bending of the fragments due to the action of the IPMF. This is, in fact, opposite to the approach taken by Gerasimova and Zatsepin. They neglected the effect of the IPMF and assumed that the distribution function of shower core separations was given only by the angular distribution of the fragments Lorentz-transformed to the Earth rest frame.

We consider a spherical volume surrounding the Earth and extending up to $r_{\max }=4$ AU. The photon density is too low and the fragment deflections too large for any significant contribution to come from outside this region. A grid is constructed giving the fragment separation at Earth for a parent Fe nucleus interacting at any point inside the volume. As we are more interested in an upper limit than in an accurate calculation, we further assume 
that all of the cross-section goes into single nucleon emission producing, as daughters, both a Mn nucleus and a proton.

To present the results we have chosen a polar coordinate system analogous to the galactic coordinate system. The reference system is centered on the Earth and its equatorial plane coincides with the ecliptic plane. The Sun is located at the origin of both longitude $\phi$ and latitude $\theta$. Latitudes are positive to the North, while longitudes are positive to dusk and negative to dawn. Distances, in AU, are measured outwards from the Earth.

In figure 3 we show core separation distribution functions for three particular directions on the sky: noon $\left(\phi \sim 1.3 \mathrm{deg}\right.$ - i.e., a $5 \mathrm{R}_{\odot}$ perihelion $)$, midnight $(\phi \sim-180 \mathrm{deg})$, and mid-afternoon $(\phi \sim 45 \mathrm{deg})$. For these plots the incident primaries are Fe nuclei of total energy $E=6.3 \times 10^{17} \mathrm{eV}$. This energy was chosen to be near the maximum of the fragmentation cross section for interaction with $\epsilon \sim 1 \mathrm{eV}$ photons (a typical energy of the solar radiation field photon). The three curves are proportional to the number of Gerasimova-Zatsepin events coming from each direction, and have been normalized such that the frequency is 1 for the smaller separation $\delta$ arriving from the noon-side. It can be seen that the effect of the IPMF is much larger than that arising from the transverse separation of the fragments leaving the interaction: at 1 AU the angular spread of the fragments gives a separation of about $15 \mathrm{~km}$. In fact the separation produced by the magnetic field is so large that the possibility of observing both partners of a disintegration process is rather limited at $\gamma \sim 10^{7}$ for any existing detector.

The mean free path of a nucleus against photodisintegration is given by:

$$
\frac{1}{\lambda(l)}=\int_{0}^{\infty} n(l, \epsilon) \times \sigma_{f r g}\left\{2 \gamma \epsilon \cos ^{2}\left[\frac{\alpha(l)}{2}\right]\right\} \times 2 \cos ^{2}\left[\frac{\alpha(l)}{2}\right] d \epsilon
$$

where $l$ is the coordinate along the path of the nucleus, $\epsilon$ is the photon energy in the rest 
frame of the Sun, $\sigma_{f r g}$ is the fragmentation cross-section specified previously, and $\alpha$ is the angle between the propagation directions of the nucleous and the photon, the latter being taken as a heliocentric radiovector.

Therefore, defining $\Phi_{\infty}$ as the unperturbed incoming cosmic ray flux at the external border, $r_{\max }=4 A U$, and $\Phi_{G Z}$ as the flux of Gerasimova and Zatsepin fragment pairs, the relative $\mathbf{G Z}$ flux is:

$$
\eta_{G Z}=\frac{\Phi_{G Z}}{\Phi_{\infty}}=1-\exp \left[-\int_{r_{\max }}^{r_{\min }} \frac{d l}{\lambda(l)}\right]
$$

where $r_{\text {min }} \sim 0.02 \mathrm{AU}$ is adopted as the inner spherical surface up to which the integration is carried out.

Figures $4 \mathrm{a}$ and $4 \mathrm{~b}$ show all sky maps of the ratio $\eta_{G Z}=\Phi_{G Z} / \Phi_{\infty}$, i.e., of the fraction of Gerasimova-Zatsepin events among the incoming cosmic ray flux. The Sun is at the center of each figure, and the ecliptic plane runs horizontally through the middle of the figure $(\theta=0)$. The shaded function in the background is $\eta_{G Z}$, while the contour lines indicate the medians of the separation between the cores of correlated showers for each direction in the sky. Note that the labels on the separation contours of figure 4a are logarithmic, while those in figure $4 \mathrm{~b}$ are linear. Both figures are calculated for monoenergetic Fe nuclei at $E=6.3 \times 10^{17} \mathrm{eV}$ (roughly the maximum of the photodisintegration cross section). In Figure 4a no account has been taken of any acceptance effects such as would be imposed in practice by a detector system: every event is counted irrespective of the separation of the showers.

In Figure 4b we show only those shower pairs for which the cores are separated by $\delta<10 \mathrm{~km}$. Such a separation is relevant for the AGASA array (Chiba et al. 1992) which has a collecting area of $100 \mathrm{~km}^{2}$. From these two figures it can be clearly seen that high 
values of $\eta_{G Z}$ only be obtained in the day-side in the vicinity of the Sun. Furthermore, large values of $\eta_{G Z}$ arise only when no cut-off in separation is considered: for any real instrument of finite size (figure 4b) the flux is several orders of magnitude smaller. In fact, so severe is this effect that when the separation is taken into account( figure $4 \mathrm{~b}$ ), the maximum $\eta_{G Z}$ is obtained on the night side of the earth around midnight. In other words, the small number of photons nightward of the earth is more than compensated by the high deflection on the day side which limits the effective integration volume to regions very near the Earth. The decrease of the effective integration volume on the day side can easily be seen by the change in symmetry of the function $\eta_{G Z}$ in going from figure $4 \mathrm{a}$ to $4 \mathrm{~b}$. In the first case there is axial symmetry around the Sun because most of the events originates in its vicinity. In figure $4 \mathrm{~b}$, when only small $\delta$ events are accepted, they originate very near the Earth, and therefore there is symmetry with respect to the ecliptic plane, revealing the planar spiral topology of the IPMF in the neighborhood of the Earth.

The low values of $\eta$ imply very low GZ fluxes. To make an estimate we assume that all nuclei above $E \sim 6 \times 10^{17} \mathrm{eV}$ are of iron and that the integral flux is $3.8 \times 10^{-12}$ $m^{-2} s^{-1} s r^{-1}$ ( a value based on Fly's Eye and Haverah Park data). Adopting an average value of $\eta_{G Z}=10^{-6}$ (figure $4 \mathrm{~b}$ ) we find the rate of GZ events to be $\sim 0.01$ per year on $100 \mathrm{~km}^{2}$ (the AGASA area). Even for the detectors of the Auger Observatory (3000 km² per site) only $\sim 0.3$ events per year with less than $10 \mathrm{~km}$ separation would land on the array. These rates are much too small for detection in the case of AGASA and for the Auger observatory the events will be difficult to identify within the background as the shower from the nucleonic fragment is likely to trigger only one detector because of the $1.5 \mathrm{~km}$ separation planned for the array. Our estimate of the rate is several orders of magnitude less than that made in the original examination of this effect in part because of the magnetic deflections but also because modern estimates of the intensity at $6 \times 10^{17} \mathrm{eV}$ are about 15 lower that believed in the late 1950s. 
The situation does not get better at higher energies even for the planned large area detectors such as the Auger project and the proposed OWL satellites. The latter experiment involves a pair of twin satellites observing extensive showers in the upper atmosphere from the outer space. The advantage of such an experiment is the huge exposure area, $10^{5}-10^{6}$ $\mathrm{km}^{2}$. The disadvantage from the point of view of GZ events is, however, that OWL observes the night side of the atmosphere where the expected $\eta_{G Z}$ is much smaller. Figures $5 \mathrm{a}$ and $5 \mathrm{~b}$ illustrate the situation at higher energies: $E=3 \times 10^{18} \mathrm{eV}$ and $E=3 \times 10^{19} \mathrm{eV}$ respectively. In both cases a maximum separation $\delta \max =1000 \mathrm{~km}$ is used, representative of a $10^{6} \mathrm{~km}^{2}$ experiment like OWL. The medians of the separations are conveniently smaller, but the GZ fluxes are again too low to be of practical use. Adopting an integral intensity of $2 \times 10^{-14}$ $\mathrm{m}^{-2} \mathrm{~s}^{-1} \mathrm{sr}-1$ and $\eta_{G Z}=10^{-7}$ gives only 0.06 events per year for an area on $10^{6} \mathrm{~km}^{2}$. Hence the GZ flux is too low to make detection practical. Furthermore, at very high energies the separation between the cores is so small $\left(\delta \sim 5 \mathrm{~km}\right.$ on the night side at $3 \times 10^{19} \mathrm{eV}$, or even $\delta \sim 0.5 \mathrm{~km} \sim 10^{20} \mathrm{eV}$ ) that confusion might arise between the signals associated with showers.

\section{Conclusions}

We have re-analyzed the proposal of Gerasimova and Zatsepin (1960) of using pairs of correlated showers, originating in the photodisintegration of heavy nuclei interacting with the solar radiation field, as a mass-spectrometric technique.

We consider a simple but realistic model of the interplanetary magnetic field to demonstrate that the magnetic deflection dominates the distribution function of core separations. The interactions with the solar radiation field inside a sphere of $4 \mathrm{AU}$ around the Earth are calculated for incoming Fe nuclei under the simplifying assumption of single 
nucleon emission. The results are presented as all-sky maps, highlighting the considerable anisotropy of the solution.

From our calculations it is apparent that the events arising from this very beautiful idea are too infrequent to be of use in any real experiment, either in operation or currently proposed, as a mass measuring technique.

This work was done with the partial support of the Brazilian agency FAPESP. 


\section{Figure Captions}

Figure 1: (a) Total magnitude of the interplanetary magnetic field and dynamo and ring current components as function of heliocentric distance over a plane parallel to the ecliptic plane and located at $\mathrm{z}=0.01 \mathrm{AU}$. (b) absolute value of the cylindrical components of the total magnetic field; the signs of the several components are indicated above the corresponding curves.

Figure 2: Iron nuclei photodisintegration cross sections. See text for details.

Figure 3: Core separation distribution functions. All the curves have the same normalization and can therefore be compared directly. each curve corresponds to a particular direction in the sky, as indicated in the respective labels, inside a solid angle of $\sim 2 \times 10^{-3}$ sr.

Figure 4: Fraction of GZ events, $\eta_{G Z}$, for Fe nuclei at $E=6.3 \times 10^{17} \mathrm{eV}$ (a) regardless of the separation $\delta$ between correlated EAS and (b) only for $\delta<10 \mathrm{~km}$. The contour lines indicate the median of the separation $\delta$, and are labeled logarithmically $\left(\log _{10}\right)$ in (a) and linearly in (b). The Sun is at the center of the image, while midnight is at the left and right borders of the figures.

Figure 5: As in figure 4, but for (a) $E=3 \times 10^{18} \mathrm{eV}$ and (b) $E=3 \times 10^{19} \mathrm{eV}$. The maximum separation allowed is in both cases $\delta_{\max }=10^{3} \mathrm{~km}$, and so this corresponds to a hypothetical experiment with an effective area of $\sim 10^{6} \mathrm{~km}^{2}$. The contour lines are labeled linearly with the median of $\delta$ inside each solid angle. 


\section{REFERENCES}

Akasofu S.-I., Gray P. C. and Lee L. C., 1980, Planet. Sp. Sci. 28, 609.

Chiba N. et al., 1992, Nucl. Inst. Mem. A, 311, 338.

Danos M. and Fuller E. G., 1965, Ann. Rev. Nucl., 15, 29.

Gerasimova N. M. and Zatsepin G. T., 1960, Soviet Phys. JETP, 11, 899.

Ginzburg V. L. and Syrovatsky S. I., 1964, in 'The Origin of Cosmic Rays', p127, Pergammon Press.

Hayward E., 1970, NBS Monog. No. 118.

Puget J. L., Stecker F. W., Bredekamp, J. H., 1976, Astrophys. J., 205, 638.

Watson A. A., 1997, 25th ICRC, rapporteur paper.

Zatsepin G. T., 1951, Dokl. Akad. Nauk SSSR 80, 577. 

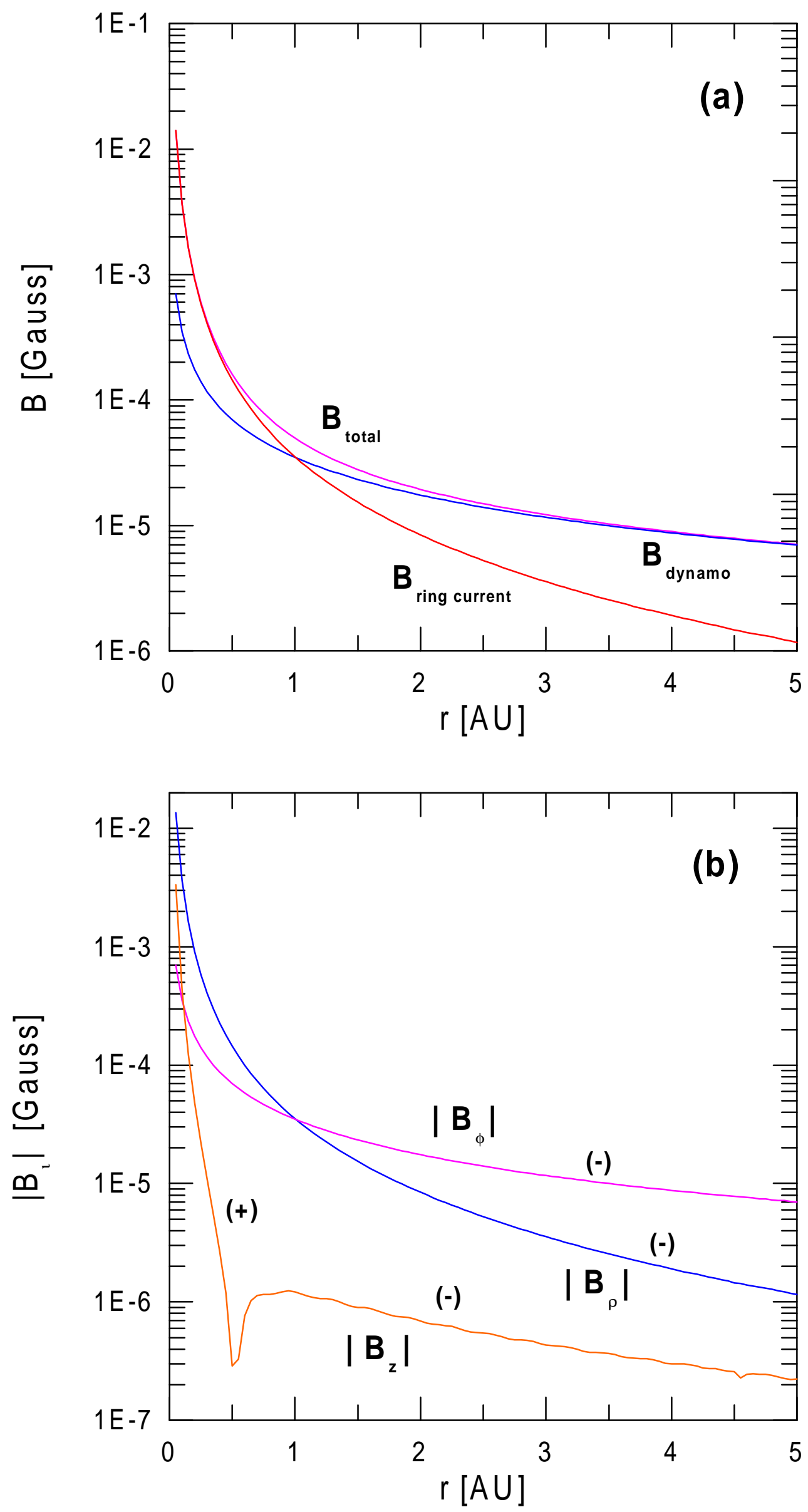

Figure 1.a \& 1.b 


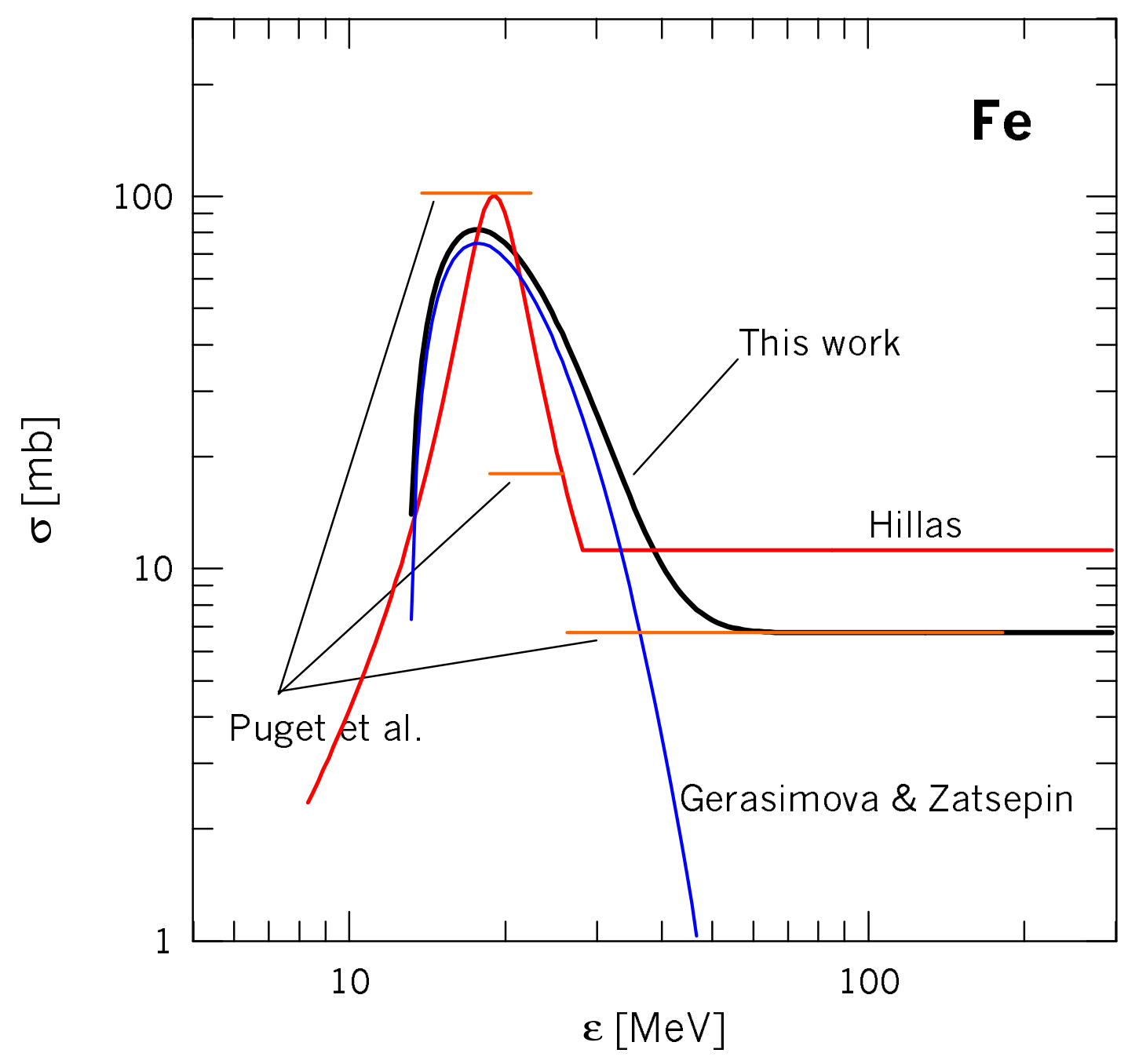

Figure 2 


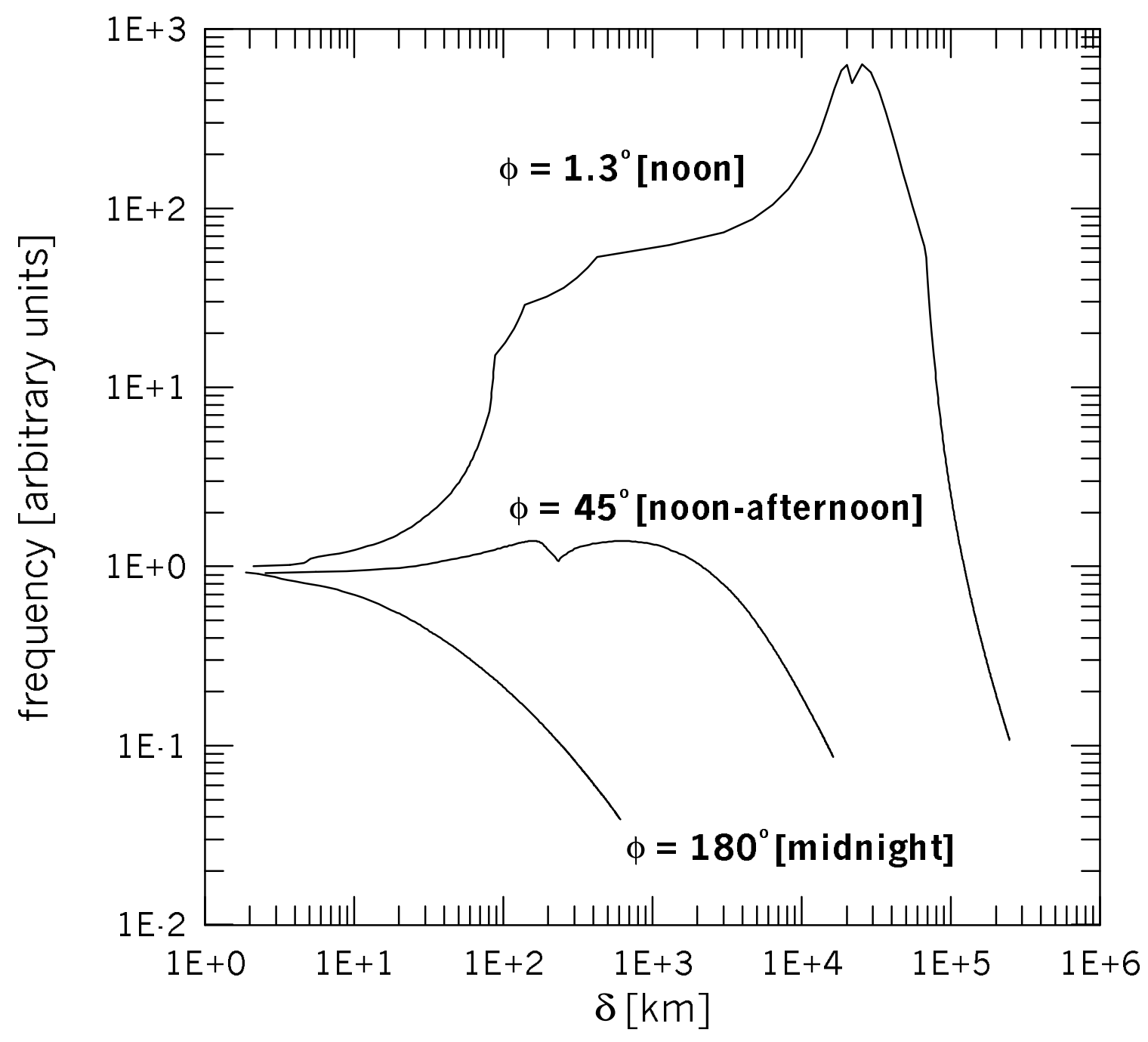

Figure 3 
This figure "figure4a.jpg" is available in "jpg" format from: http://arxiv.org/ps/astro-ph/9808033v2 
This figure "figure4b.jpg" is available in "jpg" format from: http://arxiv.org/ps/astro-ph/9808033v2 
This figure "figure5a.jpg" is available in "jpg" format from: http://arxiv.org/ps/astro-ph/9808033v2 
This figure "figure5b.jpg" is available in "jpg" format from: http://arxiv.org/ps/astro-ph/9808033v2 